University of Nebraska - Lincoln

DigitalCommons@University of Nebraska - Lincoln

8-15-2005

\title{
Direct studies of domain switching dynamics in thin film ferroelectric capacitors
}

\author{
Alexei Gruverman \\ University of Nebraska-Lincoln, agruverman2@unl.edu \\ B. J. Rodriguez \\ North Carolina State University, Raleigh, brian.rodriguez@ucd.ie \\ C. Dehoff \\ North Carolina State University, Raleigh \\ J. D. Waldrep \\ North Carolina State University, Raleigh
}

A. I. Kingon

North Carolina State University, Raleigh, angus_kingon@brown.edu

See next page for additional authors

Follow this and additional works at: https://digitalcommons.unl.edu/physicsgruverman

Part of the Physics Commons

Gruverman, Alexei; Rodriguez, B. J.; Dehoff, C.; Waldrep, J. D.; Kingon, A. I.; Nemanich, R. J.; and Cross, J. S., "Direct studies of domain switching dynamics in thin film ferroelectric capacitors" (2005). Alexei Gruverman Publications. 16.

https://digitalcommons.unl.edu/physicsgruverman/16

This Article is brought to you for free and open access by the Research Papers in Physics and Astronomy at DigitalCommons@University of Nebraska - Lincoln. It has been accepted for inclusion in Alexei Gruverman Publications by an authorized administrator of DigitalCommons@University of Nebraska - Lincoln. 


\section{Authors}

Alexei Gruverman, B. J. Rodriguez, C. Dehoff, J. D. Waldrep, A. I. Kingon, R. J. Nemanich, and J. S. Cross 


\title{
Direct studies of domain switching dynamics in thin film ferroelectric capacitors
}

\author{
A. Gruverman, ${ }^{\text {a) }}$ B. J. Rodriguez, C. Dehoff, ${ }^{\text {b) }}$ J. D. Waldrep, \\ A. I. Kingon, and R. J. Nemanich \\ Department of Materials Science and Engineering and Department of Physics, North Carolina State \\ University, Raleigh, North Carolina 27601
}

\section{J. S. Cross}

Fujitsu Laboratories Ltd., Device and Materials Laboratories, Atsugi, Japan

(Received 14 March 2005; accepted 23 June 2005; published online 15 August 2005)

\begin{abstract}
An experimental approach for direct studies of the polarization reversal mechanism in thin film ferroelectric capacitors based on piezoresponse force microscopy (PFM) in conjunction with pulse switching capabilities is presented. Instant domain configurations developing in a $3 \times 3 \mu \mathrm{m}^{2}$ capacitor at different stages of the polarization reversal process have been registered using step-by-step switching and subsequent PFM imaging. The developed approach allows direct comparison of experimentally measured microscopic switching behavior with parameters used by phenomenological switching models. It has been found that in the low field regime (just above the threshold value) used in the present study, the mechanism of polarization reversal changes during the switching cycle from the initial nucleation-dominated process to the lateral domain expansion at the later stages. The classical nucleation model of Kolmogorov-Avrami-Ishibashi (KAI) provides reasonable approximation for the nucleation-dominated stage of switching but is inapplicable to the slow switching stage. It has been suggested that the switching dynamics can be approximated by averaging the KAI model over a broad distribution of switching times. (C) 2005 American Institute of Physics. [DOI: 10.1063/1.2010605]
\end{abstract}

Ferroelectric random access memory (FeRAM) is one of the most promising and technologically advanced nonvolatile memory technologies that are in use today. It offers such benefits as high reading and writing speed, low power consumption, high endurance, and better scalability than other nonvolatile memories. However, transition from low-density to high-density FeRAM requires a major breakthrough in reducing its operating voltage as well as in understanding the switching dynamics in micrometer size ferroelectric capacitors. There have been numerous efforts to deduce the mechanism of polarization reversal in thin film capacitors by studying and modeling the transient switching current. The most promising results were obtained in the frame of the Kolmogorov-Avrami-Ishibashi (KAI) nucleation theory ${ }^{1-3}$ by considering this process as the phase transformation in infinite media with a random distribution of nucleation sites. The model was further modified to account for the effect of grain boundaries and finite size systems. ${ }^{4,5}$ However, the assumptions, under which those models were developed, such as constant nucleation rate, have proven difficult to verify experimentally in the case of thin film capacitors. Furthermore, experiments on nucleation dynamics in ferroelectric crystals show that in the high-field regime the domain nucleation rate is not constant but actually increases until the very end of the switching process. ${ }^{6}$ There are also indications of spatial variations in domain wall speed. ${ }^{7}$ This means that the approach postulating a constant nucleation rate might be not applicable to thin films where switching occurs mainly in the high-field range.

\footnotetext{
${ }^{a)}$ Author to whom correspondence should be addressed; electronic mail: alexei_gruverman@ncsu.edu

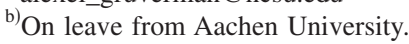

Although there are several well-documented studies of switching behavior in microscale thin film capacitors ${ }^{8-14}$ there is still a remarkable lack of information on their actual domain dynamics. In this letter, we report results of direct studies of domain switching dynamics in ferroelectric $\mathrm{Pb}(\mathrm{Zr}, \mathrm{Ti}) \mathrm{O}_{3}$ (PZT) capacitors using piezoresponse force microscopy (PFM). ${ }^{15}$ The step-by-step switching approach used in this letter opens a way for clarification of polarization reversal mechanism in thin film capacitors and direct experimental verification of the existing theoretical models of switching.

Samples used in this study are 180-nm-thick (111)oriented $\mathrm{PZT} / \mathrm{Pt}$ capacitors with $3 \times 3 \mu \mathrm{m}^{2} \mathrm{IrO}_{2}$ top electrodes. ${ }^{8}$ An experimental setup for measurements of domain switching dynamics in micrometer scale ferroelectric capacitors is described elsewhere. ${ }^{16}$ It is based on a commercial atomic force microscope (Park Scientific Instruments Autoprobe M5), equipped with Fluke PM5138 pulse generator and a Texas Instruments THS5138 evaluation module board. Voltage pulses have been applied to the PZT capacitors via Pt-coated Si cantilevers $(5 \mathrm{~N} / \mathrm{m}$ force constant, MikroMasch). The same cantilevers have been used for PFM domain imaging in the capacitors. Spatial resolution of PFM in this case was about $30 \mathrm{~nm}$. With the probing tip connected to the $3 \times 3 \mu \mathrm{m}^{2}$ capacitor, the setup can generate a voltage pulse with the rising time of about 20 ns. ${ }^{16}$

To study domain dynamics we employ the so-called step-by-step switching approach in conjunction with PFM imaging. The pulse sequence used in our studies is shown in Fig. 1. This method had been previously used at the macroscopic level in classical switching experiments on studying the correlation between domain dynamics and transient currents in ferroelectric crystals ${ }^{17}$ and later had been applied to 


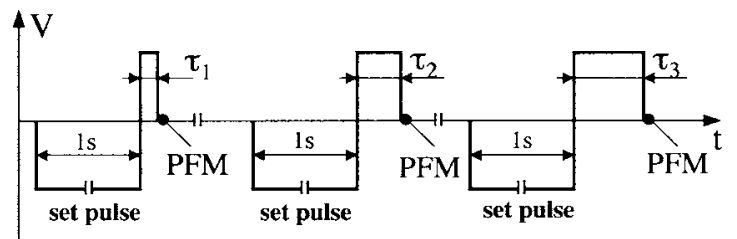

FIG. 1. Sequence of voltage pulses used to study domain dynamics using step-by-step switching.

thin films. ${ }^{18}$ In this approach, partial reversal of polarization is generated by applying a voltage pulse shorter than the total switching time $t_{\mathrm{sw}}$ and with the pulse amplitude fixed above the threshold voltage. By applying a sequence of such short pulses of incrementally increasing duration $\left(\tau_{1}<\tau_{2}<\tau_{3}\right.$ $<\ldots<t_{\text {sw }}$ ) with PFM domain imaging after each pulse, a consistent picture of domain dynamics can be obtained. At the beginning of each switching cycle, a capacitor is set into the opposite polarization state by applying a setting pulse $(-5 \mathrm{~V}, 1 \mathrm{~s})$. Since the time resolution is determined by the pulse length increment, this approach allows circumventing a problem of low time resolution in PFM. Due to the bandwidth of the pulse generator, the shortest voltage pulse that could be applied was of $220 \mathrm{~ns}$. This, in turn, determined the voltage range for the measurements. For example, for the pulse amplitude of $1.6 \mathrm{~V}$, about $70 \%$ of the total capacitor area switches within the $200 \mathrm{~ns}$ time frame. In this study, pulses in the range from $1.0 \mathrm{~V}$ (threshold value) to $1.5 \mathrm{~V}$ have been used, which means that the domain switching dynamics has been investigated in the low-field regime, where the switching times are in the order of microseconds or more.

To avoid misinterpretation of domain dynamics due to domain instability, PFM examination has been first performed at different time intervals after switching pulses. The optimum conditions for nondestructive PFM domain imaging were found at $0.4 \mathrm{~V} \mathrm{rms}(10 \mathrm{kHz})$ and $0.1 \mathrm{~V} \mathrm{dc}$ offset to account for an internal bias (imprint) in the capacitors. ${ }^{8}$ Under these imaging conditions, no difference between domain patterns recorded $\sim 2$ and $\sim 30$ min after positive pulse application has been observed indicative of domain stability.

Figure 2 shows phase and magnitude PFM images of domain configurations developing in a $3 \times 3 \mu \mathrm{m}^{2}$ capacitor at different stages of polarization reversal process. One important piece of information on domain dynamics that can be immediately extracted using this approach is related to the velocity of domain growth in forward and lateral directions. The forward growth velocity has been evaluated from analysis of the changes in the PFM amplitude that accompany domain growth through the film thickness. ${ }^{19}$ By measuring the time interval from the moment when amplitude signal starts to decrease (domain generation at the film/electrode

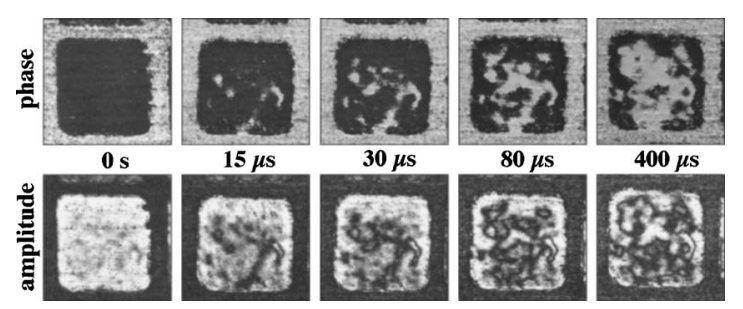

FIG. 2. PFM phase and amplitude images of instantaneous domain configurations developing in the $3 \times 3 \mu \mathrm{m}^{2}$ PZT capacitor at different stages of polarization reversal under the $1.1 \mathrm{~V}$ bias.
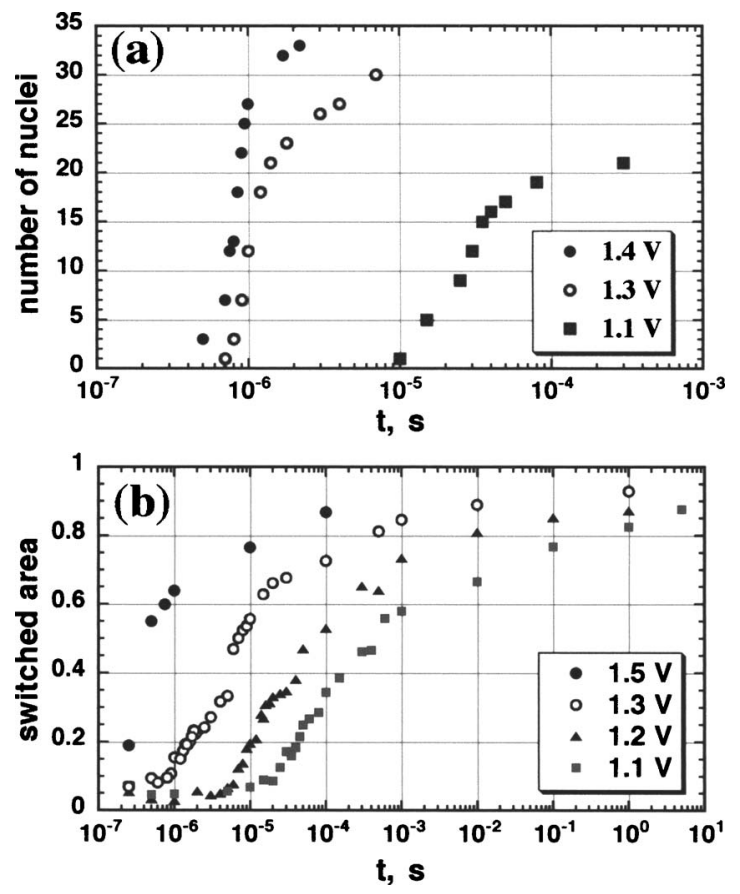

FIG. 3. (a) Time dependence of the total number of nuclei; (b) time dependence of the switched capacitor area.

interfaces) until it reaches its maximum again (domain fully penetrates the film) we were able to evaluate the domain forward velocity $v_{f}$ in the direction of the film thickness. For $V=1.4 \mathrm{~V}(E=78 \mathrm{kV} / \mathrm{cm})$ we obtained a $v_{f}$ value in the range of $0.3-0.4 \mathrm{~m} / \mathrm{s}$. Furthermore, the lateral wall velocity has been obtained from the time dependence of the domain radius as described in Ref. 20. It has been found that lateral expansion occurs with a speed that (a) fluctuates during domain growth by at least three orders of magnitude (presumably due to the domain interaction with grain boundaries or microdefects), and (b) decreases significantly before coalescing with nearby domains. The lateral wall velocity $v_{\ell}$ of an isolated domain growing in the field of $78 \mathrm{kV} / \mathrm{cm}$ was measured to be $\sim 1 \mathrm{~m} / \mathrm{s}$ and velocity drops to just several centimeters per second at a distance of $\sim 200-300 \mathrm{~nm}$ from the approaching wall of another domain. Note that due to the resolution limit it is difficult to establish whether lateral domain expansion is a result of single domain wall motion or generation of much smaller domains at close proximity to the border between oppositely polarized regions (similar to the "correlated nucleation" effect ${ }^{15}$ ). Therefore, the lateral domain growth is described in terms of the "effective" domain wall motion.

Analysis of data in Fig. 2 also allowed direct study of the mechanism of polarization reversal. It has been found that the early stages of switching ${ }^{21}$ proceed via domain nucleation $^{22}$ and then continues primarily via lateral domain expansion. From the image analysis of the instant domain patterns, the time dependencies of the total number of nucleated domains and the switched area have been obtained (Fig. $3)$. It can be seen that while nucleation ends well before the end of the switching process, the fraction of capacitor area switched due to nucleation increases with voltage. In addition, the nucleation rate does not stay constant throughout the switching-after reaching a maximum it gradually decreases to zero. However, there is not enough data to determine the exact time dependence of the nucleation kinetics. 


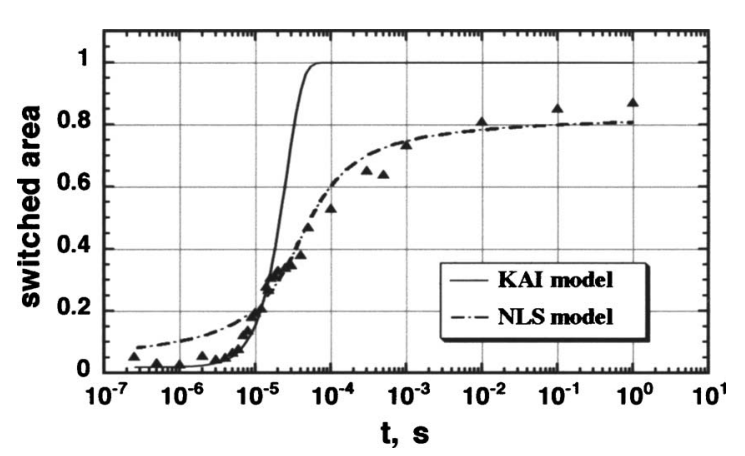

FIG. 4. Fitting of the time dependence of the switched area for the $1.2 \mathrm{~V}$ bias by the KAI function $q(t)=1-\exp \left(-\left(t / t_{0}\right)^{n}\right)$ (solid line) and by the NLS model (see Ref. 27) (dashed line).

In spite of this nonsteady process, the KAI model provides reasonable approximation for the nucleation-dominated stage of switching. Figure 4 shows a representative fitting of the experimental data by $q(t)=1-\exp \left(-\left(t / t_{0}\right)^{n}\right)$ with $n=2$, where $t_{0}$ is the characteristic switching time and $n$ is the dimensionality of the growing domains. ${ }^{23}$ However, the KAI model cannot adequately describe the slow part of the switching curve - a limitation that had been pointed out by some early reports. ${ }^{24}$ On the other hand, the nucleation limited switching (NLS) mechanism ${ }^{25-27}$ provides much better fitting for the slower part of the switching curve. However, the observed microscopic mechanism for the slow stage of polarization reversal seems to contradict the mechanism postulated in the NLS models. The experimentally observed fluctuations of the wall velocity by three orders of magnitude are indicative of a broad distribution of the wall mobility. Therefore, based on the present results, we suggest that approximation of the switching dynamics, at least in the lowfield range, should be made by averaging the KAI model over a broad distribution of parameter $t_{0}$, since it is based on the experimentally verified microscopic mechanism of domain dynamics. It should be mentioned that in the high-field range the switching mechanism might be quite different, such as being dominated by nucleation throughout the switching process. Also, for polycrystalline films, finite grain size should be taken into account as the presence of the grain boundaries may cause a significant difference in switching behavior compared to epitaxial films. ${ }^{28}$ Registration of domain dynamics using the step-by-step switching along with the switching current recording can clarify this problem. In addition, the developed approach can address the question of whether there are slow switching regions in the capacitors that do not contribute to the switching current signals in FeRAM devices. This work is under way.

In summary, the step-by-step switching approach in conjunction with the PFM imaging of domain configurations allowed direct studies of domain switching dynamics in 3 $\times 3 \mu \mathrm{m}^{2}$ ferroelectric capacitors. It has been found that in the low-field range (just above the threshold) the polarization reversal process consists of a fast switching initial part dominated by nucleation and a slow switching part due to the lateral domain growth. Analysis of the PFM data showed that the lateral wall velocity fluctuates during switching and is affected by the presence of the nearby domain walls. The nucleation-dominated stage of polarization reversal can be well approximated by the KAI model, which, however, does not provide adequate description of the slow switching stage. It has been suggested that the switching dynamics should be approximated by averaging the KAI model over a broad distribution of parameter $t_{0}$.

The authors acknowledge financial support from the National Science Foundation (Grant No. DMR02-35632) and Fujitsu Laboratories Ltd.

${ }^{1}$ Y. Ishibashi and Y. Takagi, J. Phys. Soc. Jpn. 31, 506 (1971).

${ }^{2}$ M. Avrami, J. Chem. Phys. 7, 1003 (1939).

${ }^{3}$ A. N. Kolmogorov, Izv. Akad. Nauk USSR; Ser. Math. 3, 355 (1937).

${ }^{4}$ H. M. Duiker and P. D. Beale, Phys. Rev. B 41, 490 (1990).

${ }^{5}$ V. Shur, E. Rumyantsev, and S. Makarov, J. Appl. Phys. 84, 445 (1998).

${ }^{6}$ A. Gruverman, N. Ponomarev, and K. Takahashi, Jpn. J. Appl. Phys., Part 1 33, 5536 (1994).

${ }^{7}$ H. Yu, V. Gopalan, J. Sindel, and C. A. Randall, J. Appl. Phys. 89, 561 (2001).

${ }^{8}$ A. Gruverman, B. J. Rodriguez, R. J. Nemanich, A. I. Kingon, J. S. Cross, and M. Tsukada, Appl. Phys. Lett. 82, 3071 (2003).

${ }^{9}$ S. Tiedke, T. Schmitz, K. Prume, A. Roelofs, T. Schneller, U. Kall, R. Waser, C. Ganpule, V. Nagarajan, A. Stanishefsky, and R. Ramesh, Appl. Phys. Lett. 79, 3678 (2001).

${ }^{10}$ I. Stolichnov, E. Colla, A. Tagantsev, S. S. N. Bharadwaja, S. Hong, N. Setter, J. Cross, and M. Tsukada, Appl. Phys. Lett. 80, 4804 (2002).

${ }^{11}$ J. Li, B. Nagaraj, H. Liang, W. Cao, C. H. Lee, and R. Ramesh, Appl. Phys. Lett. 84, 1174 (2004).

${ }^{12}$ S. Hong, E. L. Colla, E. Kim, D. V. Taylor, A. K. Tagantsev, P. Muralt, K. No, and N. Setter J. Appl. Phys. 86, 607 (1999).

${ }^{13}$ S. Prasertchoung, V. Nagarajan, Z. Ma, R. Ramesh, J. S. Cross, and M. Tsukada, Appl. Phys. Lett. 84, 3130 (2004).

${ }^{14}$ I. Stolichnov, L. Malin, E. Colla, A. K. Tagantsev, and N. Setter, Appl. Phys. Lett. 86, 012902 (2005).

${ }^{15}$ Nanoscale Characterization of Ferroelectric Materials: Scanning Probe Microscopy Approach, edited by M. Alexe and A. Gruverman (Springer, Berlin, 2004).

${ }^{16}$ C. Dehoff, B. J. Rodriguez, A. I. Kingon, R. J. Nemanich, A. Gruverman, and J. S. Cross, Rev. Sci. Instrum. 76, 023708 (2005).

${ }^{17}$ E. Fatuzzo and W. J. Merz, Ferroelectricity (North-Holland, Amsterdam, 1967).

${ }^{18}$ O. Lohse, S. Tiedke, M. Grossmann, and R. Waser, Integr. Ferroelectr. 22, 123 (1998).

${ }^{19}$ J. Woo, S. Hong, N. Setter, H. Shin, J.-U. Jeon, Y. E. Pak, and K. No, J. Vac. Sci. Technol. B 19, 818 (2001).

${ }^{20}$ T. Tybell, P. Paruch, T. Giamarchi, and J.-M. Triscone, Phys. Rev. Lett. 89, 097601 (2002).

${ }^{21}$ For the range of voltages used in these experiments the switching time extends over several decades up to several seconds.

${ }^{22}$ We use the term "nucleation" to describe emergence of new antiparallel domains within the original domain as they appear in the PFM images.

${ }^{23}$ Here we assumed that the switched capacitor area measured in PFM is proportional to the switching charge measured by the conventional Merz technique.

${ }^{24}$ O. Lohse, M. Grossmann, U. Boettger, D. Bolten, and R. Waser, J. Appl. Phys. 98, 2332 (2001).

${ }^{25}$ D. J. Jung. M. Dawber, J. F. Scott, L. J. Sinnamon, and J. M. Gregg, Integr. Ferroelectr. 48, 59 (2002).

${ }^{26}$ X. Du and I.-W. Chen, Mater. Res. Soc. Symp. Proc. 493, 311 (1998).

${ }^{27}$ A. Tagantsev, I. Stolichnov, N. Setter, J. Cross, and M. Tsukada, Phys. Rev. B 66, 214109 (2002).

${ }^{28}$ Y. W. So, D. J. Kim, T. W. Noh, J.-G. Yoon, and T. K. Song, Appl. Phys. Lett. 86, 092905 (2005). 